\title{
LIGA TRANSDISCIPLINAR DE TERAPIA INTENSIVA DA UNIVERSIDADE ESTADUAL DO SUDOESTE DA BAHIA (LITTI - UESB): FORMAÇÃO, AÇÕES, PREOCUPAÇÕES E DESAFIOS
}

\author{
Weskley Ramos Nascimento ${ }^{1}$
}

\begin{abstract}
RESUMO
Este artigo tem como objetivo descrever o processo de formação, os atuais e os futuros desafios na criação da Liga acadêmica Transdisciplinar de Terapia Intensiva da Universidade Estadual do Sudoeste da Bahia (LITTI - UESB), uma das primeiras ligas transdisciplinares de terapia intensiva no cenário nacional. $\mathrm{O}$ projeto tem um caráter extensionista continuado por essência, uma vez que visa a integração dos discentes aos serviços de terapia intensiva da região. Trata-se de um estudo de relato de experiência, contendo a descrição das ações e estratégias para a implantação da LITTI - UESB no campus de Jequié, no ano de 2010, e suas principais ações, integrando o eixo ensino e serviço. A partir do programa de mestrado da Sociedade Brasileira de Terapia Intensiva (SOBRATI), surgiu a vontade do docente Rodrigo Santos Queiroz de fundar uma liga acadêmica na UESB. Diante disso, os discentes Weskley Ramos e Yennydy Moura auxiliaram na formação e criação da LITTI. Dentre as principais realizações extensionistas da liga estão: inserção dos membros na Unidade de Terapia Intensiva (UTI) do Hospital Geral Prado Valadares (HGPV) e realização da estatística descritiva do perfil epidemiológico dessa instituição; aprovação de um membro, em primeiro lugar, no programa de
\end{abstract}

\footnotetext{
${ }^{1}$ Acadêmico do curso de Fisioterapia da UESB, $8^{\circ}$ semestre. Representante discente do Departamento de Saúde. Diretor de Relações Públicas da Liga Transdisciplinar de Terapia Intensiva (LITTI-UESB). Bolsista do Projeto PET-SAÚDE-JEQUIÉ. Presidente do Rotaract Club Jequié - gestão 2011-2012. E-mail: weskley_nascimento@hotmail.com

\begin{tabular}{l|l|l|l|l}
\hline Revista Extensão \& Cidadania & Vitória da Conquista & v. 1, n. 1 & p. 159-168 & jan./jun. 2013 \\
\hline
\end{tabular}
}


residência em Fisioterapia Pneumofuncional do Estado da Bahia (2011) e mais três aprovações na residência da Incor em São Paulo; realização de palestras sobre quatro grandes temas em terapia intensiva; promoção dos discentes em congressos. Como proposta futura, está sendo organizado um projeto de intervenção para a melhoria da qualidade de assistência da UTI do HGPV. A LITTI é uma ideia inovadora no cenário nacional, promissora no seu caráter transdisciplinar e extracurricular, que garante aos discentes um raciocínio construtivo, teórico e prático sobre os tópicos relacionados à área de terapia intensiva e estimula a formação de novas ligas nas faculdades e universidades brasileiras.

Palavras-chave: Liga Acadêmica. LITTI. Transdisciplinaridade.

\section{ABSTRACT}

This article aims to describe the process of training the current and future challenges in creating the league disciplinary academic intensive care at the State University of Southwest Bahia (LITTI - UESB), one of the first leagues disciplinary intensive care in the national. A project that has a character essentially continued by extension, since it aims at the integration of students with intensive care services in the region. This study is an experience report containing a description of actions and strategies to implement the LITTI UESB on the campus of UESB Jequié in 2010, and its main actions integrating the shaft teaching and service .. From the Masters program of the Brazilian Society of Intensive Care (SOBRATI), was the will of the teacher Rodrigo Queiroz Santos of founding an academy in UESB league. Given that the students Weskley Yennydy Moura Ramos and assisted in the formation and creation of LITTI. Among the main achievements extension of the league are: Insertion of the members in the Intensive Care Unit (ICU) of Hospital General Prado Valadares (HGPV) and performing the descriptive statistics of the epidemiological profile of the institution; Approval of a member first in the residency program Respiratory Therapy in the State of Bahia (2011) and three more approvals at the residence of Incor in Sao Paulo, Lectures on four major themes in intensive care, promotion of students in congress. As future proposals, is being organized an intervention project to improve the quality of care in the ICU of HGPV. The LITTI is an innovative idea on the national scene, promising disciplinary in character and extracurricular, guaranteeing students a constructive reasoning, theoretical and practical training on topics related to the field of intensive care, should be stimulated the formation of new leagues in colleges and Brazilian universities.

Keywords: Academic League. LITTI. Transdisciplinarity. 


\section{Introdução}

As Ligas Acadêmicas (LA) é um espaço que oportuniza atividades científicas, culturais e sociais para a comunidade interna e externa. No Brasil, as primeiras ligas foram fundadas em um momento de grande tensão político-social, correspondente aos "anos de chumbo". Nesse período, associações estudantis passaram a discutir a essência do ensino universitário, o direcionamento e a aplicabilidade dos avanços técnico-científicos.

Em 30 anos de redemocratização, mudanças profundas na sociedade, na abordagem de Atenção à Saúde e nas formações curriculares aconteceram labutadas às ligas acadêmicas. Para orientar e organizar a criação das ligas, a Direção Executiva Nacional dos Estudantes de Medicina (DENEM) elaborou um guia de criação de ligas que seria um tipo de "receita" a ser adaptada às demandas locais (LETUFPE, 2007).

$\mathrm{Na}$ carta constitucional de 1988, foi elaborado o princípio da indissociabilidade entre ensino, pesquisa e extensão, o qual fortaleceu o papel das LA. Criada em 1961 e atualizada em 1996, a Lei de Diretrizes e Bases da Educação Nacional (LDB), que é a lei orgânica e geral da educação brasileira, propiciou maior autonomia dos órgãos estaduais, o que diminuiu a centralização do poder do MEC. Além disso, definiu o papel da educação superior na prática e na formação acadêmicas. A LDB privilegia o estímulo ao conhecimento dos problemas do mundo presente de forma descentralizada e estabelece a autonomia para as universidades elaborarem seus currículos dos cursos de graduação.

O Parecer no 1.133, da Câmara de Educação Superior (CES), do Conselho Nacional de Educação (CNE), reforça a necessidade da articulação entre educação superior e saúde, com o intuito de melhorar a qualidade da formação acadêmica. O resultado prático é a oportunidade de vislumbrar uma comunidade assistida por um serviço de alta qualidade e a consignação de uma relação comunidade 
$\mathrm{X}$ futuros profissionais em potencial. Deste modo, as atividades de extensão, como as ligas acadêmicas, devem pesquisar e fazer com que os estudos acadêmicos cheguem rapidamente à comunidade, por meio da prática profissional (TORRES et al., 2008).

Configurado esse raciocínio, espera-se que as ligas apresentem momentos nos quais os alunos possam atuar junto à comunidade como agentes transformadores, associando o ensino e a pesquisa com a prática extensiva e que este ato amplifique a atividade e o conhecimento do acadêmico (TORRES et al., 2008).

Apesar do desenvolvimento acadêmico e da importância extracurricular das ligas, não se pode olvidar os riscos à formação dos futuros profissionais. A falta de orientação pedagógica e a inadequação da supervisão de docente são alguns pontos que merecem destaque antes da criação de uma liga acadêmica em uma instituição universitária. Segundo Hamamoto Filho et al. (2010 apud TAQUETTE et al., 2003), uma das consequências dos estágios práticos sem supervisão adequada é a aprendizagem de conceitos e técnicas errados e a incorporação de condutas antiéticas à prática profissional. Estudos atuais mostram que existe uma verdadeira preocupação para que as ligas não se transformem em meras atividades de iniciação científica e de assistência, afastando de sua função primordial de extensão universitária, com atividades de prevenção de doenças e promoção da saúde (TORRES et al., 2008).

\section{Formação da Liga Transdisciplinar de Terapia Intensiva da Universidade Estadual do Sudoeste da Bahia (LITTI - UESB)}

O conteúdo ministrado no curso de graduação não é a única forma de aprendizado do estudante. Nesse mesmo raciocínio, o professor não é o conhecedor de todas as coisas. Em busca de um aprendizado diferencial foi que surgiu a Liga Transdisciplinar de Terapia Intensiva (LITTTI), uma associação de acadêmicos dos cursos de Fisioterapia, Enfermagem, Medicina e Psicologia, sem fins lucrativos, tendo como princípio servir à comunidade acadêmica. 
A formação se iniciou a partir de um curso na Sociedade Brasileira de Terapia Intensiva (SOBRATI), quando o docente Rodrigo Santos Queiroz analisou que no estado da Bahia não existia nenhuma liga que fosse Transdisciplinar e que a Universidade Estadual do Sudoeste da Bahia (UESB) possuía potencial para implementar as ligas acadêmicas, porém, necessitava de um elo entre os acadêmicos e a ideia de inovadora, até então. Dessa forma, foram convidados os discentes Weskley Ramos e Yennydy Moura para que auxiliassem no processo de fundação e implementação.

Pela falta de modelo de ligas transdisciplinares no Brasil, a Liga Estudantil (LE) na UESB iniciou com formato experimental, como grupo de trabalho/estudo entre os estudantes de fisioterapia. Por meio dessas reuniões os anseios entre os alunos foram aumentando e a transdisciplinaridade, que é uma ideia piagetiana, foi cultivada, a fim de formar um grupo mais conciso e original. Segundo Cunha (2003), o termo transdisciplinaridade é definido como: "transgressão das fronteiras entre as disciplinas, diferenciando-o da pluridisciplinaridade e da interdisciplinaridade". O transdisciplinar deve ser entendido, pois, como em transição a outras disciplinas.

Posteriormente, o grupo convidou outros estudantes que perpassam pela área de Terapia Intensiva e que estavam regularmente matriculados na UESB. Assim, discentes dos cursos de Medicina e Enfermagem começaram a participar e contribuir nas reuniões. Em seguida, ampliou-se a iniciativa para que, além dos estudantes da UESB, fizessem parte também os estudantes de Psicologia da Faculdade de Tecnologia e Ciências (FTC). Criado o caráter da liga, o contexto era obedecer à LDB e formalizar, diante dos órgãos competentes, a criação da LITTI. Dessa forma, foi emitido um documento de reconhecimento à UESB, ao Departamento de Saúde da instituição (DS), Colegiado de Fisioterapia, Colegiado de Enfermagem, Colegiado de Medicina, Hospital Geral Prado Valadares (HGPV) e à SOBRATI.

A primeira formação da LITTI foi composta por um coordenador docente, por dois coordenadores discentes e grupos 
de trabalho (Secretaria, Epidemiologia, Produção Científica, Oficiais Emergencistas e Residência em Terapia Intensiva).

Atualmente, a LITTI é composta por uma diretoria executiva prevista por estatuto que apresenta: um presidente, um vice-presidente, um tesoureiro, um diretor de relações públicas e o professor coordenador. Além disso, os grupos de trabalho permanecem, porém, passíveis de modificações ou eliminações provenientes da diretoria e em assembleia geral.

Para ser admitido na instituição, é necessário passar por um processo seletivo que consta de uma prova objetiva com 12 questões de temas definidos em assembleia, sendo três questões referentes ao estatuto. Anteriormente à prova, a LITTI possui o compromisso de apresentar um curso introdutório, que explana os objetivos, deveres e ações do grupo. Podem se inscrever para o processo de seleção os alunos regularmente matriculados nos cursos de Graduação em Fisioterapia ( $3^{\circ}$ ao $9^{\circ}$ semestre); Graduação em Enfermagem $\left(4^{\circ}\right.$ ao $8^{\circ}$ semestre); Graduação em Medicina ( $2^{\circ}$ ao $11^{\circ}$ semestre); Graduação em Psicologia pela FTC - campus de Jequié ( $3^{\circ}$ ao $9^{\circ}$ semestre). Em caso de empate, será realizada uma entrevista com os candidatos, a fim de classificá-los.

\section{Atividade extensionista da LITTI}

Com pouco mais de um ano de existência, a LITTI tem desenvolvido trabalhos juntamente com as suas parceiras. Com o HGPV, podemos destacar a proposta extensionista de inserção dos membros na UTI em forma de observação e também para a realização da estatística descritiva do perfil epidemiológico da unidade; essa tarefa é de fundamental importância, pois leva ao conhecimento dos gestores e da comunidade os principais indicadores epidemiológicos da UTI.

Fora desenvolvido ainda uma metodologia de discussões na unidade de terapia intensiva.

$\mathrm{Na}$ instituição ESB realizamos, nesse semestre, palestras sobre quatro grandes temas em terapia intensiva (Fisiopatologia 
da Hipertensão Intracraniana, Assistência ao paciente na UTI, portador de distúrbios respiratórios, Consenso Brasileiro de Suporte Hemodinâmico, Suporte Básico de Vida); além disso, é uma constante observar a equipe LIT'TI nos eventos de cunho regional, estadual, nacional e até mesmo internacional. É salutar lembrar que no ano de 2010 tivemos também destaque no cenário estadual após a aprovação de um membro, em primeiro lugar, no programa de residência em Fisioterapia Pneumofuncional do Estado da Bahia. No final de 2011, despontamos no cenário nacional após a entrada de três membros no Instituto do Coração da USP (INCOR).

\section{Preocupações e desafios da LITTI}

Por se tratar de uma Liga Acadêmica transdisciplinar, ou seja, uma ideia inovadora no cenário nacional, não fica difícil entender que muitos obstáculos serão observados. Pode-se começar pela dificuldade de encontrar modelos para orientar e facilitar algumas indagações. Dentre tantas, destacam-se: além da universidade, qual outro órgão precisa ser submetido à LIT'TI no cenário nacional para tornar reconhecido? Como orientar todos os cursos de graduação em torno de pontos comuns na UTI?

As LA são essencialmente médicas. Segundo a Liga Acadêmica de Medicina intensiva (LIGAMI, 2011): “As Ligas Acadêmicas são entidades de reconhecida relevância na participação da educação médica no meio acadêmico". Percebe-se que existe uma organização em âmbito nacional e, além disso, os estudantes entendem a importância das atividades extracurriculares.

É fundamental que exista uma base orçamentária, ou seja, antes de saber quanto será preciso arrecadar, é necessário tomar conhecimento de quanto será gasto. No que diz respeito a orçamento, Chiavenato (2003) explica que são planos relacionados com dinheiro, receita ou despesa, dentro de um determinado espaço de tempo. Conforme suas dimensões e efeitos, os orçamentos são planos estratégicos quando envolvem a entidade como uma totalidade e abrangem um período 
longo. Esse orçamento descriminado dos gastos é importante para promover a apresentação do projeto da liga a possíveis apoiadores e patrocinadores. Porém, a LITTI apresenta como forma de arrecadação, somente, a promoção de cursos, simpósios, congressos e doações. A falta de verba é um fator complicador no desenvolvimento de estudos mais atuais, em que há carência do material para realização das práticas.

O conhecimento do termo transdisciplinaridade ainda traz muitas dúvidas e esse talvez seja o medo de algumas profissões e/ ou profissionais de ingressarem nessa nova dinâmica de grupo. Com certeza, esse "novo" termo precisa ainda ser debatido nos diversos setores e com os mais diversos especialistas. Segundo o Conselho Federal de Medicina (CFM), no uso das atribuições que lhe confere a Lei $\mathrm{n}^{\circ} 3.268$, de 30 de setembro de 1957, regulamentada pelo Decreto no 44.405, de 19 de julho de 1958, há a necessidade de uma melhor definição das atividades profissionais típicas e privativas de cada categoria profissional, dos seus limites, das relações entre as atividades limítrofes e das relações de cada uma delas com a Medicina, por ser, de todas, a mais antiga e a de campo mais amplo de atuação, uma vez que interage com todas as outras (CFM, 2001).

\section{Metodologia}

Trata-se de estudo de relato de experiência, com a descrição das ações e estratégias para implantação da LITTI - UESB, campus Jequié, nos anos de 2010-2011. As discussões e experiências são relatadas mediante as reuniões ocorridas na Clínica Escola de Fisioterapia e no Auditório Administrativo da UESB, toda sexta-feira, das $18 \mathrm{~h}$ às $19 \mathrm{~h}$, tendo como foco a Terapia Intensiva no cenário Regional, Estadual e Nacional.

\section{Resultado}

A LITTI é uma ideia inovadora no cenário estadual e nacional, promissora no seu caráter transdisciplinar, a qual garante aos discentes um raciocínio construtivo em um ambiente, que a cada dia vem sendo mais explorado na saúde pública, que é a resolução 
dos problemas, com enfoque de todos os âmbitos profissionais. Com o desenvolvimento da LITTI, a sociedade regional, inserida no contexto da UTI, ganha um aliado no desenvolvimento da formação acadêmica e profissional; além disso, a população ganha membros sedentos na conjuntura ensino-aprendizado, a fim de auxiliar na resolução do problema do doente.

O HGPV tornou um parceiro nessa nova relação de trabalho com a liga acadêmica, sendo uma das poucas no cenário estadual a apresentar dados concisos e factuais da UTI. Além disso, o hospital se converteu em um "laboratório vivo" para os estudantes, o que possibilita um conhecimento diferenciado na grade universitária.

A LITTI inova nos diálogos entres os estudantes, apresentando um grupo de residência (grupo de estudos que se prepara para as principais residências no Brasil), que discorre em reuniões os mais diversos assuntos: saúde pública, sistema cardiorrespiratório, aparelhos da UTI dentre outros.

\section{Conclusão}

No panorama nacional/estadual, a LIT'TI apresenta-se ousada em relação às ligas acadêmicas, que, na sua maioria, são médicas. Apesar das diversas preocupações e desafios, os acadêmicos vêm aprendendo mais sobre a UTI, o que possibilita aos próprios tornarem futuros profissionais capacitados para entrarem nesse ambiente.

\section{Referências}

CHIAVENATO, Idalberto. Administração de recursos bumanos: fundamentos básicos. 5. ed. São Paulo: Atlas, 2003.

CUNHA, S. S. O Manifesto da Transdisciplinaridade, por Basarab Nicolescu: um breve resumo. Salvador: Nepol, 2003. 
HAMAMOTO FILHO, Pedro Tadao et al. Normatização da abertura de ligas acadêmicas: a experiência da Faculdade de Medicina de Botucatu. Rev. bras. educ. med., Rio de Janeiro, v. 34, n. 1, mar. 2010. Disponível em: <http:/ / www.scielo.br/scielo.php?script $=$ sci_arttext\&pid $=$ S010055022010000100019\&lng=en\&nrm=iso $>$. Acesso em: 28 mar. 2011.

CONSELHO FEDERAL DEMEDICINA. Resolução CFMn $n^{\circ} .627 / 2001$. 2001. Disponível em: <http://pnass.datasus.gov.br/documentos/ normas/89.pdf>. Acesso em: 19 abr. 2011.

LIGA ACADÊMICA DE MEDICINA INTENSIVA (LIGAMI). Acadêmicos interessados em Medicina Intensiva. 2011. Disponível em: $<$ http://www.amib.org.br/detalhe/noticia/academicos-interessadosem-medicina-intensiva/>. Acesso em: 28 mar. 2011.

LIGA DE EMERGÊNCIA E TRAUMA DA UNIVERSIDADE FEDERAL DE PERNAMBUCO (LETUFPE). Ligas Acadêmicas no Brasil. Recife, 2007. Disponível em: <http://www.grupos.com.br/ blog/letufpe>. Acesso em: 17 mar. 2011.

MOURA et al. Estatuto da Liga Transdisciplinar de Terapia Intensiva. Jequié, 2011.

SILVA, M. I; VASCONCELOS, C. B. Health Promotion at Organizational Space: The Study of Interdisciplinary Team for Call Center's Workers. The FIEP Bulletin, v. 79, p. 54-57, 2009.

TORRES, A. R. et al. Ligas Acadêmicas e formação médica: contribuições e desafios. Interface (Botucatu), Botucatu, v. 12, n. 27, dez. 2008. Disponível em: <http://www.scielo.br/scielo.php?script $=$ sci_ arttext\&pid $=$ S1414-32832008000400003\&lng $=$ en\& $\mathrm{nrm}=$ iso $>$. Acesso em: 28 mar. 2011. 